\title{
Submitted: Immersion pulmonary oedema in a triathlete - a diagnostic Accepted: challenge in sports cardiology
} 31.03.2021

Published: 16.08.2021

\begin{abstract}
Keywords
immersion pulmonary oedema, swimming-induced pulmonary oedema, triathlon, dyspnoea, sildenafil
\end{abstract}

\author{
Anna Sobieszek¹, Marcin Konopka1', Marek Cacko ${ }^{2,3}$, \\ Marek Kuch ${ }^{4}$, Wojciech Braksator ${ }^{1}$ \\ ${ }^{1}$ Department of Sports Cardiology and Noninvasive Cardiovascular Imaging, Medical \\ University of Warsaw, Warsaw, Poland \\ ${ }^{2}$ Department of Diagnostic Imaging, Mazovia Brodno Hospital, Warsaw, Poland \\ ${ }^{3}$ Department of Nuclear Medicine, Medical University of Warsaw, Warsaw, Poland \\ ${ }^{4}$ Department of Cardiology, Hypertension and Internal Diseases, Medical University of Warsaw, \\ Poland, Mazovia Brodno Hospital, Warsaw, Poland
}

Correspondence: Anna Sobieszek, Mazowiecki Szpital Bródnowski, ul. Kondratowicza 8, 03-242 Warszawa; tel.: 2232658 24, e-mail: anias_15@o2.pl

DOI: $10.15557 / \mathrm{JoU} .2021 .0041$

\begin{abstract}
Immersion pulmonary oedema, also referred to as swimming-induced pulmonary oedema, is a form of pulmonary oedema which usually occurs during swimming in cold water. The condition is most common in triathlon athletes; it was first reported in military divers. The main symptoms include acute dyspnoea, cough, and occasionally haemoptysis, which usually subside within approximately 48 hours. The pathomechanism is not fully understood, but oedema is suspected to be due to an increased systemic vascular resistance that overloads the left ventricle. The diagnostic process can be challenging and require multiple stages to rule out a number of other possible conditions. In view of the circumstances in which incidents typically occur, immersion pulmonary oedema poses an immediate life threat to individuals involved in selected forms of physical activity, where survival is often determined by appropriate training of medical services.
\end{abstract}

\section{Case report}

We present the case of a 48-year-old female triathlete who had a sudden attack of severe dyspnoea during an amateur sports competition. The incident happened in a lake (air temperature: 21C, water temperature: 17C) in August 2020. After the triathlete swam a distance of 200-300 m she developed severe shortness of breath, which made it impossible to continue competing.

The woman was pulled out of the water by emergency services; she categorically denied aspiration of water. Despite the cessation of physical activity the complaints persisted, and the triathlete experienced considerable weakness and confusion. Crackling sounds could be heard above the lungs. The level of capillary saturation was $84 \%$. Oxygen therapy, intravenous furosemide and inhaled salbutamol were administered, and the athlete was transported to hospital.
The electrocardiogram was normal. Chest radiography (X-ray) revealed areas of marked parenchymal thickening in the lower and middle lung fields bilaterally (Fig. 1A). Laboratory tests showed significantly elevated levels of cardiac troponin $\mathrm{T}$ (cTnI) (1048 ng/l; normal range <16 ng/l), B-type natriuretic peptide (BNP) (6001.6 pg/ml; normal range $<125 \mathrm{pg} / \mathrm{ml})$, and D-dimer (DD) (2555 ng/ml; normal range $<550 \mathrm{ng} / \mathrm{ml})$. The results of a molecular test (real-time polymerase chain reaction, RT-PCR) and an assay to detect SARS-CoV-2 antibodies were negative. Angiographic evaluation of the thoracic arteries ruled out pulmonary embolism and thoracic aortic pathologies. At the same time, diffuse ground-glass opacities were seen in the pulmonary parenchyma (Fig. 2A).

The crackles over the lower lung fields persisted on the following day of hospitalisation. Lung ultrasound (LUS) was performed, showing the presence of B-lines (Fig. 2B and Fig. 2C). Echocardiography revealed a 

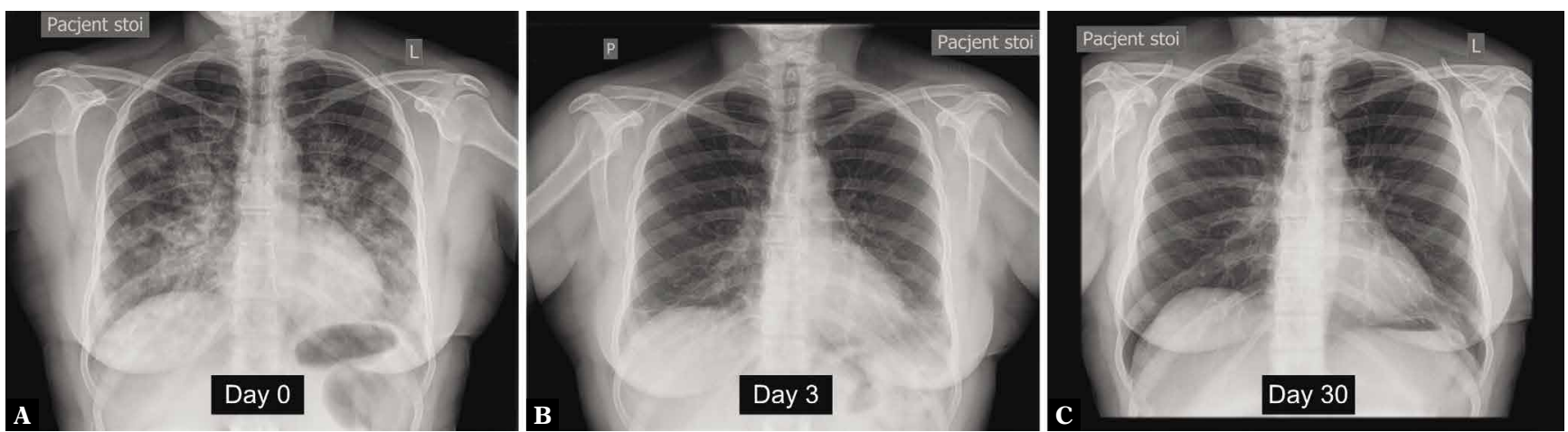

Fig. 1. Chest radiography findings at three different time points during the period of patient care. A. Examination performed in the emergency department (Day 0) - marked parenchymal changes involving the middle and lower lung fields. B. Another day of hospitalisation (Day 3) - clear regression of parenchymal lesions. C. Long-term evaluation after the original incident (Day 30) - complete resolution of parenchymal lesions
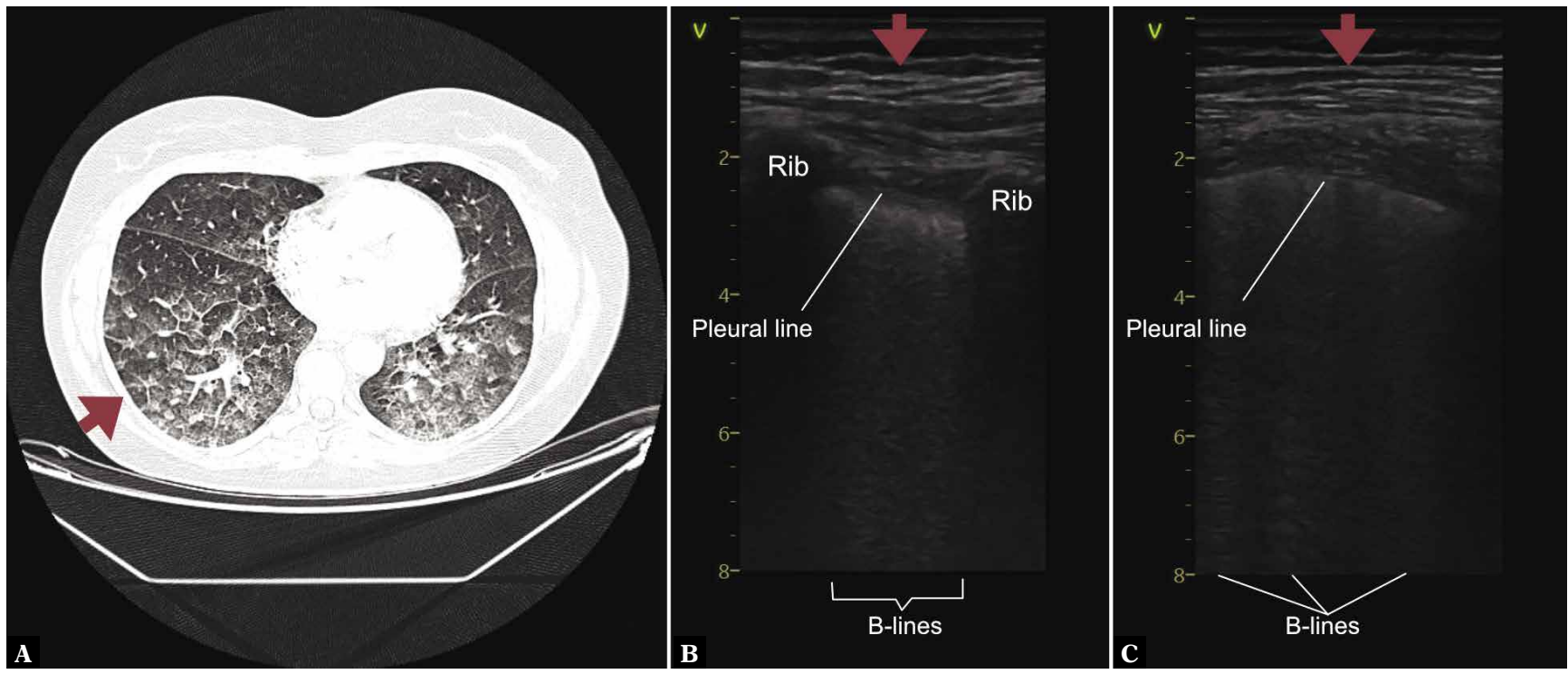

Fig. 2. Chest CT scan dedicated to the assessment of pulmonary parenchyma - high-resolution computed tomography (HRCT) (A). Comparative concurrent lung ultrasound examination performed with a linear transducer $(\mathbf{B}, \mathbf{C})$. A. CT scan revealing extensive ground-glass parenchymal opacities. B, C. Lung ultrasound showing diffuse B-lines. The red arrowhead shows the approximate location where the linear was applied. A clear correlation is seen between B-lines and ground-glass opacities

moderately enlarged right ventricular cavity - RVA/ LVA (right/left ventricular analysis) $=1.03$ (Fig. 3A). The left ventricular systolic and diastolic function was normal (ejection fraction, EF 65\%). The parameters of the right ventricular systolic function were also within the normal range: TAPSE (tricuspid annular plane systolic excursion) $22 \mathrm{~mm}$; there was a change in the right ventricular area (fractional area change, FAC; 42\%). In addition, global longitudinal strain was measured $(-24.5 \%)$ (Fig. 3B).

On the third hospital day, the patient did not report any complaints. Radiographic examination revealed regression of parenchymal lesions (Fig. 1B). The levels of cTnI (42 ng/l), BNP (1018 ng/ml), and DD (202 ng/ml), rapidly normalised. 24-hour Holter monitoring (ECG and blood pressure) found no abnormalities.

The patient was reassessed 30 days after the incident. Laboratory tests showed complete normalisation of
cTnI (0 ng/l), BNP (97.8 pg/ml), and DD (104 ng/ml) levels. The patient's chest X-ray showed no evidence of any abnormalities (Fig. 1C). Cardiac echocardiography revealed a significant improvement in the parameters of right ventricular morphology and function. In addition, RVA/LVA = 0.79 (previously: 1.03) (Fig. 3C). An improvement was observed in TAPSE - $26 \mathrm{~mm}$, FAC $52 \%$, and right ventricular free-wall global longitudinal strain (-29.37\%) (Fig. 3D).

Considering the fact that consequences of ischaemic heart disease are among the most common causes of death in athletes aged $>35$ years, the diagnostic work-up was extended in this direction. Cardiac stress scintigraphy (single-photon emission computed tomography, SPECT) was done, without any abnormalities (Fig. 4 and Fig. 5).

Based on the overall clinical presentation and the results of additional examinations, the patient was diagnosed with immersion pulmonary oedema (IPO), also referred to as 

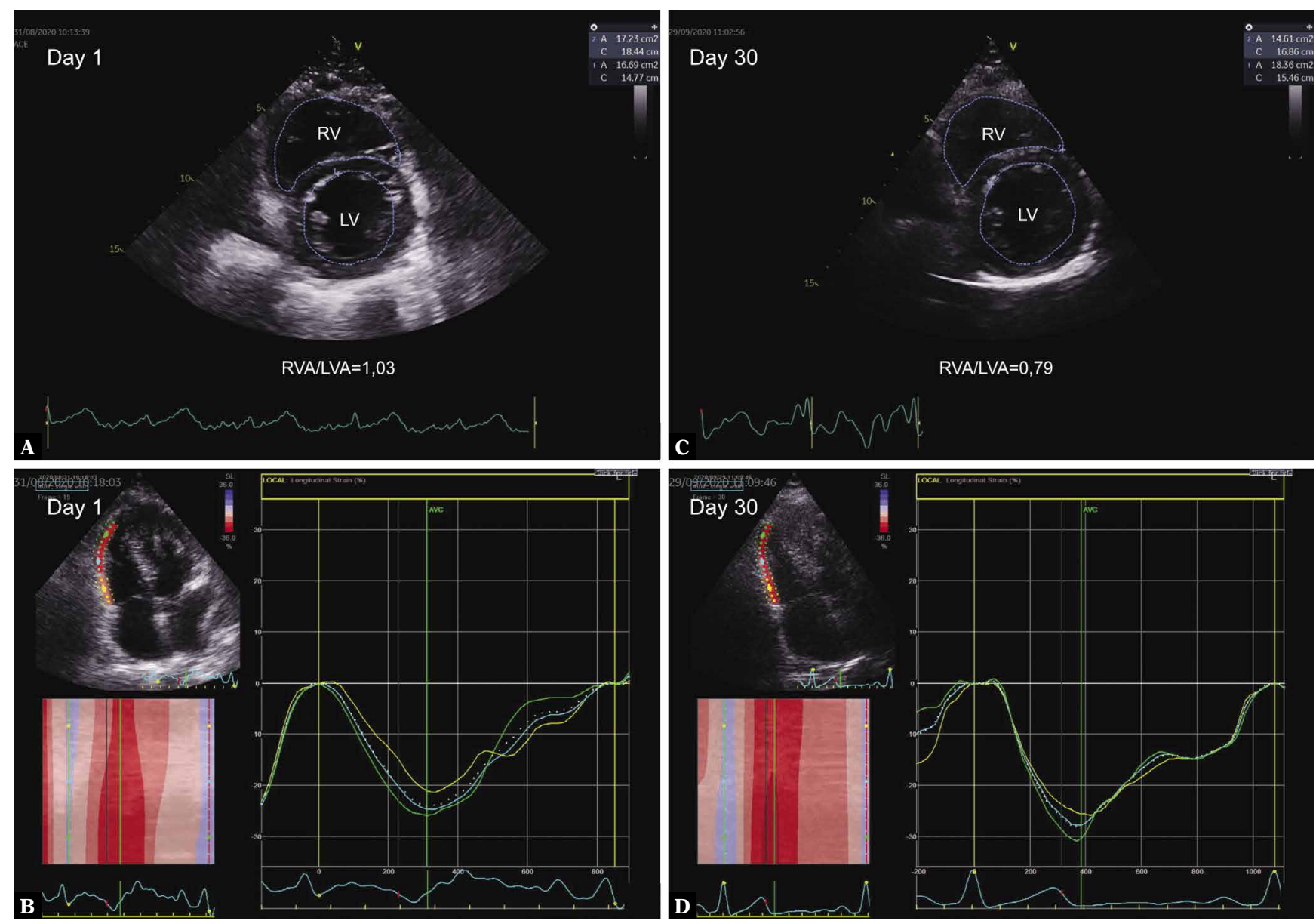

Fig. 3. Echocardiographic evaluation. A, C. Evaluation of the right and left ventricular relations at different time points during the period of patient care; a marked change in the ratio between the right and the left ventricle areas (RVS/LVA) over several days of follow-up (Day 1 vs Day 30) is noted. B, D. Assessment of right ventricular systolic function by tracking acoustic markers; compared to the baseline image (Day 0), there is a marked improvement in right ventricular free-wall longitudinal strain (Day 30). RV - right ventricle; LV-left ventricle; $R V A$ - right ventricle area; LVA - left ventricle area

swimming-induced pulmonary oedema (SIPO), with features of acute right ventricular heart failure.

\section{Discussion}

Triathlon is a physically demanding multi-sport discipline included in the group of endurance sports. It consists of three components: swimming (750-3800 m), cycling (13$112 \mathrm{~km})$, and running $(5-42 \mathrm{~km})$.

Regular physical activity is known to reduce overall mortality and the risk of cardiovascular death. At the same time, however, inappropriate types of physical activities may lead to serious health effects. The risk of sports-related death is $0.3-2.1$ cases per 100,000 population/year; in young athletes ( $<35$ years of age) it is 0.3 cases per 100,000 population/year, while in older athletes it rises to 3 cases per 100,000 population/year. The risk is higher in men. In younger athletes, $<35$ years of age, the main causes of death include cardiomyopathies and channelopathies, and the associated cardiac arrhythmias. In older athletes (aged $>35$ years), complications of ischaemic heart disease are the predominant type.

Compared to other sports, the risk of triathlon-related death is relatively high: 1.74 cases per 100,000 competitors (1.01 per 100,000 marathon runners). The majority of deaths in triathlon (67\%) occur during the swimming stage. This may delay medical rescue efforts, so the aetiology of death remains unclear. Among the causes, IPO/SIPO have been proposed ${ }^{(1)}$.

Immersion pulmonary oedema (IPO/SIPO) was first described in 1989 in divers experiencing cold water conditions $(<12 \mathrm{C})$. The researchers concluded that the main predisposing factors included hypertension, female gender, and cold water ${ }^{(2)}$.

The main symptoms are dyspnoea (79\% of cases), cough (71\%), less commonly haemoptysis, foamy sputum, chest pain/tightness, and wheezing. The disease is characterised by an acute onset of symptoms and their rapid resolution, usually within 48 hours $^{(3,4)}$.

Establishing the correct diagnosis can be challenging and requires a combined diagnostic approach. 


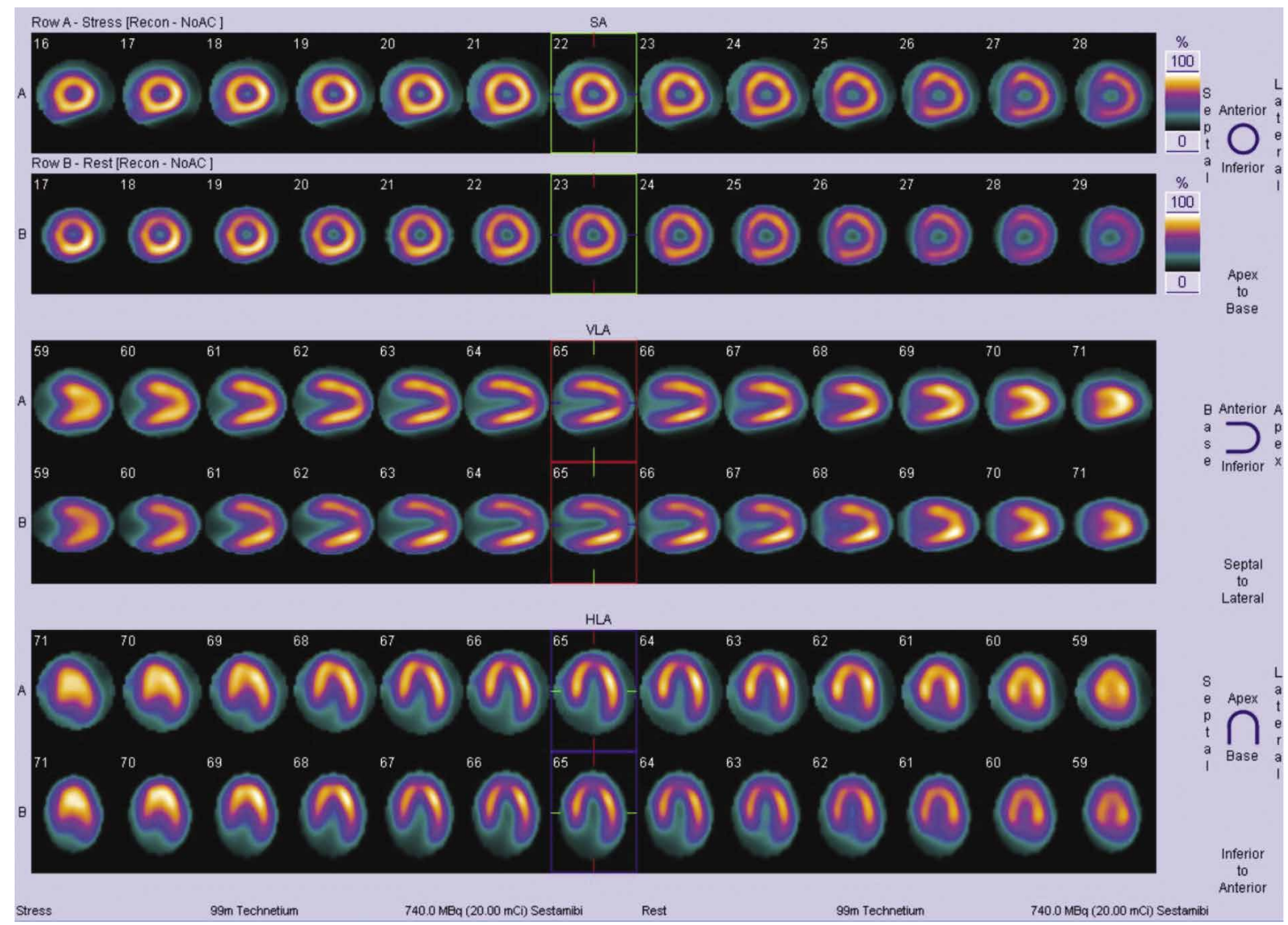

Fig. 4. Assessment of myocardial perfusion by SPECT - rest and stress tests showing no significant ischaemia. Top bar: stress-rest cross-section images in the short axis; middle bar: stress-rest horizontal cross-section images in the long axis; bottom bar: stress-rest vertical cross-section images in the short axis. SA - short axis, VLA-vertical long axis, HLA-horizontal long axis

The basic imaging examination is chest X-ray showing characteristic parenchymal densities and interstitial changes $^{(5)}$. Another diagnostically useful modality is LUS $^{(6)}$. It is capable of identifying lung oedema with high sensitivity (97\%) and specificity (95\%). The diagnosis is supported by interstitial syndrome manifested as B-predominance: the more B-lines are found, and the denser their arrangement, the greater the accumulation of fluid in the pulmonary parenchyma. "White lung" seen on LUS images is evidence for high severity of the condition ${ }^{(7)}$.

In the acute phase, treatment involves elimination of the causative factor, and supplemental oxygen therapy, bronchodilator drugs, and diuretics. Also, spontaneous resolution of symptoms has been observed in some individuals with IPO/SIPO despite no treatment.

IPO/SIPO prevention should include the identification and treatment of predisposing conditions such as arterial hypertension, atrial fibrillation, obstructive sleep apnoea, and obesity. Tight-fitting swimsuits and excessive hydration before taking part in competitions should be avoided.
There have been interesting findings in studies investigating prophylactic sildenafil as a strategy to prevent IPO/ SIPO through the drug's activity lowering pressure in the pulmonary bed. However, it is important to note that sildenafil may be dangerous in divers. Phosphodiesterase-5 inhibitors have been found to potentially contribute to an increased risk of epileptic seizures attributable to the toxic effects of oxygen on the central nervous system ${ }^{(8)}$.

In the competitor described in this case report, the diagnosis of IPO/SIPO was based on medical history and the results of additional examinations. Multi-stage diagnostic imaging also played a major role. A CT scan ruled out two serious cardiac conditions: pulmonary embolism and aortic dissection. At the same time, it confirmed parenchymal changes consistent with pulmonary oedema (previously visualised on chest X-ray).

A cardiac echo was performed to assess left ventricular morphology and function, and was normal. These findings, in combination with the patient's previous medical history (no stenocardial complaints) and electrocardiographic results (no ischaemia), helped to exclude myocardial infarction with a high level of probability. Lung 


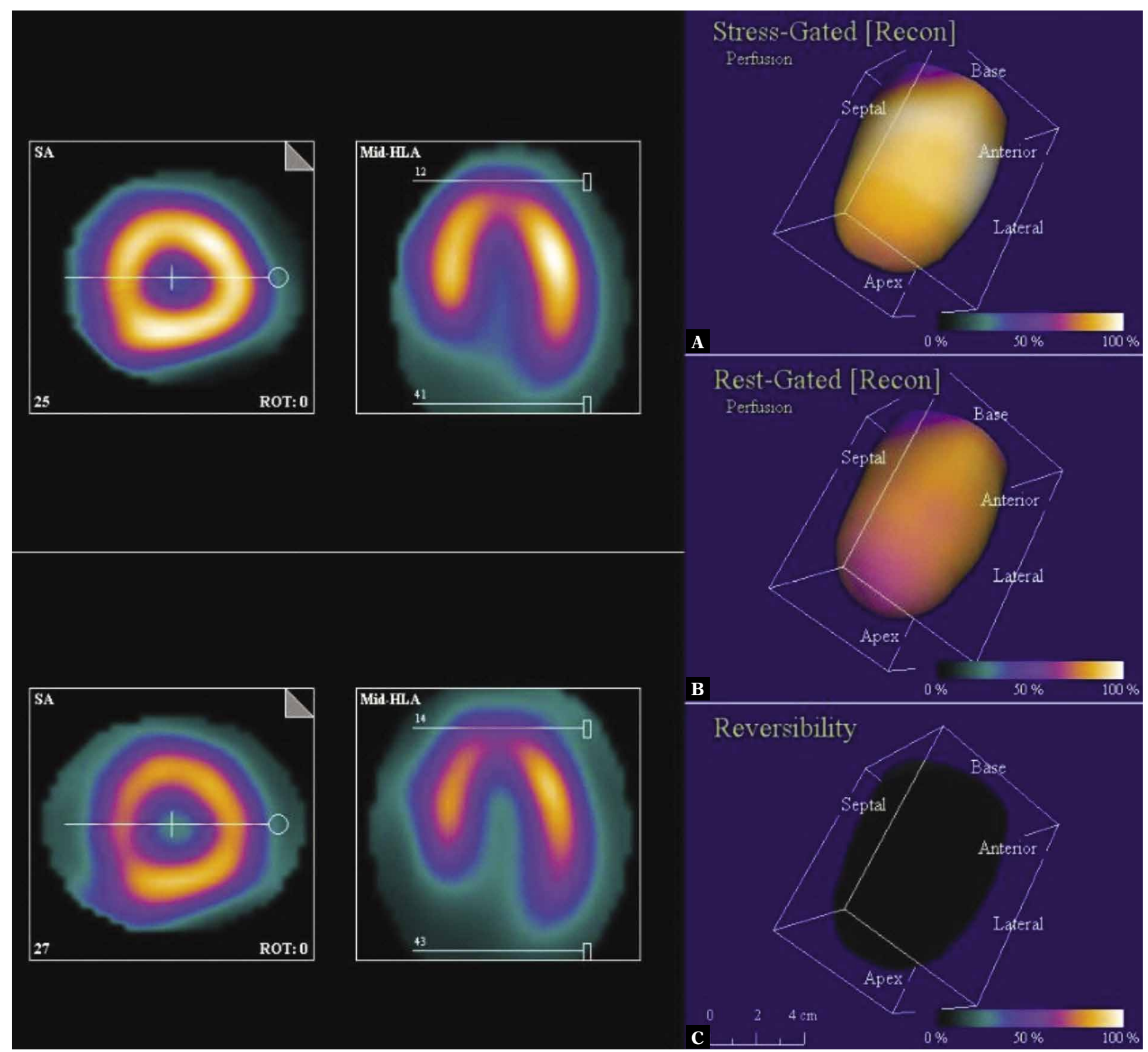

Fig. 5. Cardiac imaging using $99 m$ Tc-methoxy-isobutyl-isonitrile; 3D-Gated display. A. At stress. B. At rest. C. No difference between rest and stress tests

ultrasound, which revealed the presence of B-lines, was another modality to confirm parenchymal stasis, and was then used to monitor the patient's condition.

Very interesting insights were gained from the echocardiographic assessment of right ventricular function. In the acute phase of the disease, there was moderate enlargement of the right ventricle. The right ventricular function parameters (TAPSE, FAC, longitudinal strain) were within the normal range for the population, though they were found to be significantly impaired in that particular patient. This was revealed in the patient's reassessment performed after 30 days, which showed a change in the RVA/LVA ratio and a significant improvement in functional parameters.
The diagnostic imaging was complemented by an evaluation for ischemic heart disease performed by SPECT.

\section{Conclusions}

Immersion pulmonary oedema (IPO), also referred to as swimming-induced pulmonary oedema (SIPO) is a dangerous complication associated with the practice of certain sports, which occurs during swimming in cold water.

An important role in the diagnosis of IPO/SIPO is attributed to medical history-taking and combined diagnostic imaging 
In view of the growing interest in amateur swimming competitions (including triathlon) medical teams should be able to correctly identify IPO/SIPO in the competing athletes.

\section{References}

1. Pelliccia A, Sharma S, Gati S, Bäck M, Borjesson M, Caselli S et al.: 2020 ESC guidelines on sports cardiology and exercise in patients with cardiovascular disease. Eur Heart J 2021; 42: 17-96.

2. Moon RE, Martina SD, Peacher DF, Kraus WE: Deaths in triathletes: immersion pulmonary oedema as a possible cause. BMJ Open Sport Exerc Med 2016; 2: e000146.

3. Carter EA, Koehle MS: Immersion pulmonary edema in female triathletes. Pulm Med 2011: 261404.

4. Smith R, Ormerod JOM, Sabharwal N, Kipps C: Swimming-induced pulmonary oedema: current perspectives. Open Access J Sports Med 2018; 9: 131-137.

\section{Conflict of interest}

The authors do not declare any financial or personal links with other persons or organisations that might adversely affect the content of the publication or claim any right to the publication.

5. Grünig H, Nikolaidis PT, Moon RE, Knechtle B: Diagnosis of swimming induced pulmonary edema - a review. Front Physiol 2017; 8: 652.

6. Frassi F, Pingitore A, Cialoni D, Picano E: Chest sonography detects lung water accumulation in healthy elite apnoea divers. J Am Soc Echocardiogr 2008; 21: 1150-1155.

7. Hårdstedt M, Seiler C, Kristiansson L, Lundeqvist D, Klingberg C, Braman Eriksson A: Swimming-induced pulmonary edema diagnostic criteria validated by lung ultrasound. Chest 2020; 158: 1586-1595.

8. Martina SD, Freiberger JJ, Peacher DF, Natoli MJ, Schinazi EA, Kernagis DN et al.: Sildenafil: possible prophylaxis against swimming-induced pulmonary edema. Med Sci Sports Exerc 2017; 49: 1755-1757. 